\title{
LXXIII. Photographic reversals in spectrum photographs
}

\section{R.W. Wood}

To cite this article: R.W. Wood (1903) LXXIII. Photographic reversals in spectrum photographs, Philosophical Magazine Series 6, 6:35, 577-587, DOI: 10.1080/14786440309463058

To link to this article: http://dx.doi.org/10.1080/14786440309463058

Published online: 15 Apr 2009.

Submit your article to this journal $\pi$

Џ Article views: 3

Q View related articles $\longleftarrow$

4 Citing articles: 4 View citing articles 5 
A glance at the Plate will show that the absorption due to the presence of iron-vapour \&c. has resulted in a diminution of intensity, particularly towards the violet end of the spectrum. The phenomenon to which we wish to draw attention is independent of this effect and refers rather to the results which are observed when the spectrum is studied in detail. It is found that the simplification is purely selective in its occurrence, for whereas certain of the lines appear in this vacuum glow-spectrum with their characteristic intensity, neighbouring lines of almost equal importance in the ordinary are and self-induction spark have so greatly diminished as to be quite invisible. The effect, therefore, is in no way connected with the actual exposure of the photograph or intensity of the light.

The present experiments are not sufficiently complete to form any general theory on the effect of high pressures. They indicate, however, that work in this direction would yield results of some spectroscopical interest.

With regard to the vacuum discharge, it would be of importance to extend the work to other metals and ascertain if the simplification found in the case of iron can be considered as general.

Our sincere thanks are due to Professor Arthur Schuster for the valuable advice he has frequently given during the course of this work; we desire also to record our indebtedness to Dr. G. A. Hemsalech, who has kindly assisted in several of the experiments.

Physical Laboratories,

Owens College, Manchester.

LXXIII. Photographic Reversals in Spectrum Photographs. By R. W. Wood, Professor of Experimental Physics, Johns Hopkins University*.

[Plate XXVI.]

THEE importance of distinguishing between photographic and true reversals of lines in spectrograms has made it seem worth while to investigate with some care the conditions under which reversals due entirely to photographic action can occur. Professor Trowbridge has advanced the theory of selective reversibility of the silver salts in the sensitive film for certain wave-lengths, and it.was in part to determine whether the tendency of a line to reverse was a

* Communicated by the Author. From the Astrophysical Journal for June 1903. 
function of the wave-length, that the present investigation was undertaken.

As I showed several years ago, the Clayden effect, or the type of reversal giving rise to the phenomenon of dark lightning, results from the action of a light-shock on the plate before its exposure to diffuse light. The effect of this light shock, which must be of very brief duration, is to decrease the sensibility of the plate, resulting in a less energetic action during the subsequent illumination. I made no attempt at the time to determine the maximum duration of the light shock which would still give the Clayden reversal, but expressed the opinion that it could not exceed $1 / 10,000$ of a second. This opinion was based on a single experiment, and $I$ have since found that by a suitable adjustment of the conditions the duration may be as great as $1 / 1000$ of a second, though only a very slight trace of reversal occurs under these conditions.

It appears to me now that there are at least four different trpes of photographic reversal, or perhaps tive if we allow the chemical treatment of the plate between two exposures. As any one of these four types is liable to occur in any photographic work, when the proper conditions are fulfilled, it may be well to enumerate them at the beginning.

First Type.-The ordinary over-exposure reversal, which occurs when the plate is given three or four hundred times its normal exposure and then developed in the usual way.

Second Type.-The reversals produced by developing the plate in full lamp-light, the plate having been more or less over-exposed to begin with. This type has been extensively studied by Nipher, the results of his experiments being given in the Proceedings of the St. Louis Academy of Science.

Third Type.-This type must occur frequently, though I never remember to have seen it described. It happens when a normally or under-exposed plate is developed and then exposed to light for a minute or two before the hypo bath. The fogging, which is usually of a reddish-brown colour, does not occur on the portions of the plate where there is a developed image, and even if this image is very feeble it remains clean and almost transparent. I first noticed this effect in some photographs of spectra which sbowed strong reversals along the edges where the illumination must have been very feeble, and was unable to explain it. Further experimenting showed that it had resulted from turning up the light before the plate had been thoroughly fixed. Doubtless this effect has been described time and again in the photographic journals, but it was new to me, and may be 
to some others. Reversals of this type will be seen in fig. 6 illustrating my paper on sereens transparent to ultra-violet light*. The spectra (negative) have bright borders.

Fourth Type.-The Clayden effect, which is the type chiefly to be dealt with in the present paper. This occurs when an exposure of about 1/1000 of a second or less is given, and the plate subsequently fogged by exposure to diffuse light before development. If images of electric sparks are thrown on a plate and the plate then exposed to the light of a candle for a few seconds, the spark images will develop reversed, which is not the case if the exposure to candle-light precedes the impression of the spark inages.

This effect is shown in fig. A. A series of spark images have been gradually reversed by exposing the plate in strips

Fig. A.

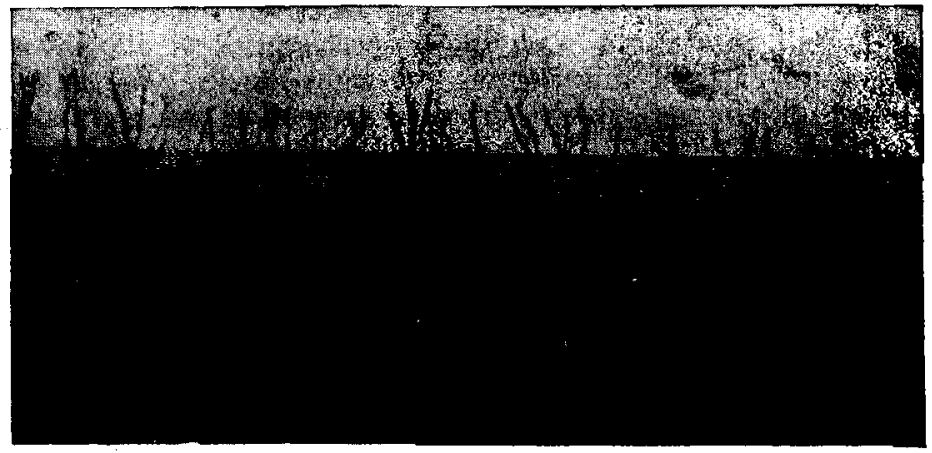

to the light of a candle. The upper strip was exposed 30 seconds, the following $20,15,10,5,2,0$ respectively.

Fifth Type.-I have found that the condition produced in the sensitive film by light shock can be imitated by treating the plate, after exposing portions of it to the action of a feeble light for a few seconds, to an oxidizing bath of bichromate of potash and nitric acid. If the plate is dried and then fogged by candle-light and developed, the previously exposed portions will come out reversed.

This effect is shown in Plate XXVI. fig.4. A series of spark images was impressed on the plate, which was then covered with a piece of black paper in which a narrow slit had been cut. A number of images of this slit were then impressed on the plate by exposure to the light of a candle. If the plate in this condition were then fogged by candle-light and

* Phil. Mng. February 1903. 
developed, the spark-images would come out reversed and the slit images not reversed. Before fogging it one end (the upper in the print) was immersed for a few minutes in a very dilute solution of bichromate of potash, slightly acidified with nitric acid. It was then dried, exposed to candle-light and developed. In the lower portion of the print we find the sparks black and the slit-images white ; in the upper portion both sets of images come out dark, the reversal of the sparks being much stronger than on the untreated portion of the plate.

In the present paper I propose to discuss the Clayden effect not only in connexion with the selective reversibility hypothesis adopted by Professor Trowbridge to explain his spectrum photographs, but also in relation to the time factor, and radiations other than light, such as the Becquerel and Röntgen rays, which are quite different in their action from light.

I shall, in dealing with the subject, speak of the initial exposure of brief duration as the light-shock. The subsequent illumination which causes the reversal of the impression of the shock, I shall call the fogging exposure.

The first subject investigated was the relation of the phenomenon to the wave-length of the light. The light shock in this case was administered by exposing the plate to the spectrum of one or more sparks between cadmium electrodes by means of a small quartz spectrograph. Even with a small diaphragm the illumination by a single spark yielded a developable image of the spectrum down to the extreme ultra-violet. Six spectra were impressed on the same plate with different sized diaphragms ; the plate was then exposed to the light of a candle for a few seconds and developed, the result being reproduced in Plate XXVI. fig. 1 . It will be seen that the lines and the continuous background come out positive in the two upper spectra, showing that if the shock is too intense no reversal takes place, a circumstance in which the Clayden effect differs essentially from ordinary reversal due to over-exposure. Professor T'rowbridge says that his reversals occur where bright lines fall on a continuous background, and considers the reversing action proportional to the product of the two effects. If his reversals are of this nature this cannot be the case, for by making one factor (the light shock) large, no trace of reversal appears. As I shall show presently this statement requires some modification, for, as we increase the intensity of the shock, we can by increasing the fogging exposure still get reversal. As I said in my previous note, it appears probable to me that in Professor 
Trowbridge's photographs of spectra of single sparks, the shock was the almost instantaneous exposure to a bright-line spectrum of exceeding brief duration, followed by an exposure to a superimposed continuous spectrum of longer duration, which may have been due to incandescence of the inner wall of the capillary tube, or to phosphorescence of the gas. By employing a tube with a bore of about $\cdot 25 \mathrm{~mm}$. I have obtained reversed lines in the blue with single discharges of a medium sized induction-coil and condenser.

The fact that the faint continuous spectrum of the spark is uniformly reversed shows that there is no selective reversibility so far as the initial light-shock is concerned.

I next endeavoured to determine whether the wave-length of the fogging-light had anything to do with the matter (in which case we should expect reversals in some parts of the spectrum and not in others), in the particular case where the fogging illumination was spread out into a spectrum as in Professor Trowbridge's photographs. Having already found that fogging the plate with $\mathrm{X}$-rays never gave reversals of spark-images, it occurred to me that possibly ultra-violet light might act in a similar manner. Having impressed a number of spark-images on a plate, it was illuminated with light of wave-length in the neighbourhood of $\lambda=23$, from a discharge between cadmium electrodes, a screen provided with a slit being placed in the focal plane of the quartz spectrograph, with the plate a short distance behind it. The sparkimages were not reversed, and it appeared at first sight as if ultra-violet light of this wave-length acted like the X-rays. I was, however, not willing to accept this conclusion without further study, since the fogging illumination in this case consists in reality of a number of feeble light-shocks, that is, it is of much briefer duration than in the case of candle-light. In the first experiment the fogging illumination by ultraviolet light was produced by the passage of perhaps a score of sparks before the slit of the spectrograph. To get a feebler illumination of longer duration I moved the next plate to a distance of about two metres from the screen and let the coil run for about a minute. The room was absulutely dark, the spark-terminals and the front of the spectrograph being covered with heavy black cloth, so that the only light that reached the plate was of the wave-length above mentioned. On this plate the spark-images which had been previously impressed were strongly reversed, showing that the time factor comes in the fogging-light as well as in the light-shock, and that ultra-violet light is as efficient as any other, if it is not of too brief duration. This appears to Phil. Mag. S. 6. Vol. b. No, 35. Non. $1903.22 \mathrm{Q}$ 
dispose of the idea of selective reversibility, at least so far as the Clayden effect is concerned.

Investigation of the Time Factor.-To determine the maximum duration of time which the light-sh sck may have and still reverse, the following method was used :-A disk of cardboard $50 \mathrm{cms}$. in diameter was mounted on the shaft of an electric motor, the speed of which could be determined by the tracing of a tuning-fork on a smoked metal plate mounted on the same shaft. Near the rim of disk a number of narrow slits were cut, varying in width from $1 \mathrm{~mm}$. to 5 mms. An arc-light was focussed on the rim by means of a large condensing-lens, the image of the crater being about half a millimetre in diameter. By driving the disk at a high rate of speed intermittent flashes of very brief duration were obtained as the slits passed across the arc's image. A short distance behind the disk a rectangular metal tube was mounted provided with a slit $1.5 \mathrm{~mm}$. wide, immediately opposite the point where the image of the arc fell on the disk. Down this tube the plate was dropped, receiving in its passage before the slit light flashes of varying duration. 'T'he plate was subsequently exposed to candle-light and developed. The images of the slit in the case of the briefest flashes were perfectly sharp, in wther cases they were broadened owing to the rapid motion of the plate. This made the interpretation of some of the records difficult, and it was found better, when working with flashes longer than 1/2000 of a second in duration, to lower the plate down the tube with a thread. A print from one of these plates is shown in fig. 2, Plate XXVI. In this case there were two $1 \mathrm{~mm}$. slits on the rim of the disk not very far apart, then a slit somewhat wider further around, and after this a still wider one. It will be seen that the slit images formed by the two very short flashes are completely reversed, while the others are only reversed on their edges.

If the plate moves during the exposure, as was the case in this photograph, it is obvious that the edges of the slitimage will receive less exposure than the centre, which accounts for the partial reversal. As the result of exposing about two dozen plates, it was found that the duration of the shock could be as long as 1/1000 of a second, and still yield reversals. It was only by carefully regulating the intensity of the fogging-light and the duration of the development that these reversals could be obtained.

When the duration is less than 1/2000 sec. reversals could be obtained without difficulty. Flashes varying in duration from $1 / 15,000$ sec. to $1 / 500$ sec. were studied, and it was 
found that as the duration of the shock was increased the reversals became weaker, the images finally failing to appear at all on the plate, notwithstanding the longer duration of the flash. On still further increasing the duration the images came out not reversed. There may be sonie connexion between the condition in which the light shocks fail to develop at all, and the zero condition of the plate described by Nipher.

The experiment in which the fogging of the plate was effected by exposure to ultra-violet light furnished by a quartz spectrograph shows that the time factor plays a role in the togging-light as well as in the light-sh'ck.

It was $\bar{f}$ und that if the fogging light was rather intense but of short duration, the image of the light-shock did not reverse; if the light was less intense, but of a little longer duration, no trace of the shock appeared, while if the light was still less intense, and of somewhat longer duration, the image came out reversed. With a suitable ratio of intensities and durations of time, it is possible to snperpose two impressions on a photographic plate, only one of which appears on development.

This effect is shown in fig. 3, Plate XXVI. A series of sparkimages of equal intensity was impressed on the plate, which was then fogged in sections, the lower strip being exposed to the light of one spark at a distance of a metre, the next to the light of four sparks at a distance of two metres, the next to nine sparks at three metres, and so on. The total amount of fogging-light was thus approximately the same in each case, though it was found that considerably greater action was produced by a large number of sparks at a $c$ msiderable distance, than by a single spark close to the plate. It will be noticed that on the third strip from the bottom there is scarcely a trace of the spark-images. The ratio of the times of duration of the shock and the fogging-light was in this case about $1: 9$. On the two strips below this one, the sparks appear not reversed, while on all of the strips above reversal has taken place.

A more careful quantitative investigation of these effects is much to be desired, with apparatus of such design that the duration and intensity of both the light-shock and fogginglight can be accurately controlled.

I am of the opinion that the result of such an investigation would be the establishment of the fact that with very brief and intense light-shocks, comparatively intense fogging-light of short duration will yield reversals, while in the case of shocks of say $1 / 1000$ sec. duration, the fogging-light must be $2 \mathrm{Q} 2$ 
feeble and of long duration in order that reversals may be obtained. My plates appear to indicate this qualitatively, but quantitative data could doubtless be obtained with suitable apparatus. As I shall show later, it is possible to administer the shock in such a manner that it comes out reversed even when the fogging-light is the flash of a single spark.

I have tried to obtain some idea of the action of the lightshock by attempting to transform its effect on the plate into an effect similar to that produced by ordinary exposure, by means of the action of various chemical agents. These experiments were all failures, but the interesting fact was ascertained that an ordinary exposure appeared to be transformed into a shock exposure by the action of a dilute bath of bichromate of potash slightly acid with $\mathrm{HNO}_{3}$. This effect is shown in fig. 4, Plate XXVI. A series of spark-images was impressed on the plate, and then a series of images obtained by illuminating the plate with the light of a candle shining through a slit in a piece of black paper. One half of the plate was then dipped into the bath, washed and dried, exposed to the light of a candle, and developed. A print from this plate is reproduced in fig. 4, Plate XXVI. On the upper portion, which was treated with bichromate, both the sparkimages and slit-images appear reversed, on the lower the latter are not reversed. This experiment merely shows that a plate which has been exposed to light in certain places and then treated to the bichromate solution, is less sensitive to the action of subsequent illumination on the spots which have previously received light. The condition may appear at first sight to be similar to that produced by a light-shock, but there is in reality probably no connexion between the two, for while light-shocks not followed by fogging can be dereloped as not reversed images, the "bichromatized images" do not develop at all unless the plate is fogged before development.

The nearest approach which $I$ have been able to make to the transformation of the effect of a light-shock into that due to ordinary exposure, is by the action of the $\mathrm{X}$-rays. It was found that spark-images could not be reversed under any circumstances if the plate was fogged by these rays instead of candle-light. To prove that the case was not analogous to the one in which ultra-violet light failed to give reversals, owing to the comparatively briet duration of the illamination, long exposures were made with the $\mathrm{X}$-ray tube at a considerable distance. Not only were reversuls nerer obtained, but it was found that after a brief exposure to the raxs fogging the plate by lamp-light failed to reverse 
the spark-images. This seemed very remarkable, for it was subsequently ascertained that X-ray images could be reversed even when produced by long exposure to feeble radiation, by subsequent exposure of the plate to lamp-light. This effect is shown in fig. 5, Plate XXVI.

The spark-images were impressed first. The plate was then exposed in vertical strips to the action of X-rays for varying lengths of time, the left-hand strip receiving the longest exposure, while the right-hand strip was not exposed at all. Following this came an exposure in horizontal strips to lamp-light, the lower strip having the longest exposure and the upper none at all. It will be seen that there is no trace of reversal in the upper left-hand corner, where the fogging is due almost wholly to X-rays, while reversed edges appear on all of the sparks in the lower right-hand corner, where the fogging was due to light: moreover, in the lower left-hand corner, where the X-ray fog preceded the light fog, the images are not reversed. On investigating the matter further, I found that shocks administered by single powerful flashes of X-rays were reversed by subsequent exposure to lamp-light. In this case, however, the time element appears to be without much influence, for images formed by long exposure to very feeble $\mathrm{X}$-radiation reverse in the same manner. This seems very remarkable, when we consider the fact that exposure to these rays changes the condition produced by light-shock in some manner, so that it is impossible to reverse it by further fogging.

The reversal by X-rays is illustrated in fig. 6. A plate

Fig. 6. was wrapped up in black paper and exposed to the radiation for several minutes, a vertical iron rod shielding

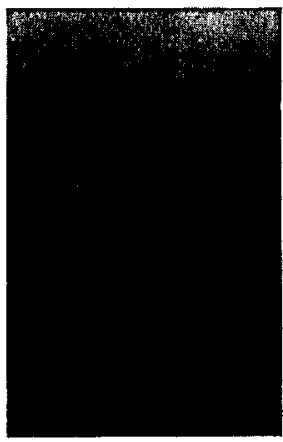
the centre strip of the plate. The plate was then exposed to lamp-light for different lengths of time in strips perpendicular to the shadow of the iron rod. On development it was found that on the end of the plate which had received the shorter exposures to light, the central strip came out lighter than the background, while on the opposite end of the plate it was darker. At a certain point near the centre of the plate, all trace of the shadow of the rod had disappeared, showing that exposure to X-rays for some time, and then to light for a certain time, produces an image no blacker than the light alone would have produced. 
I next ascertained that if the plate be exposed simultaneously to light and X-rays, the latter inhibit the action of the former. $A$ candle and an X-ray tube were set up at some little distance apart in front of a plate, the latter being much nearer the plate however, owing to its less energetic action. An iron rod mounted in front of the plate carst two shadows upon the sensitive film, one a light shadow, the other an X-ray shadow. After the double exposure the p'ate was developed, with the curious result that one shadow was darker than the background, the other lighter, showing that the light was more energetic in its action on the area screened from the X-radiation.

It may be worthy of mention that both this result and the preceding one were predicted before the actual experiments were tried. The prediction was the result of an attempt to apply Bose's strain theory of photographic ac ion * to the phenomena in question. This theory seemed rather promising at first, especially as it enabled me to predict new phenomena, but it failed to account for so many things that I was finally forced to albandon it.

The action of other stimuli wos next investigated. It luas long been known that pressure-marks on the film can be developed. I found that if the plate was fogged by lamplight before developing, the pressure-marks came out reversed. It then occurred to me try the effect of light shocks on pressure-marks, and I found to my surprise that the flash of a single spark was as effective in reversing the pressuremark as the exposure to the lamp. The pressure-marks can also be reversed by exposure to $\mathrm{X}$-rays.

As a result of numerous other experiments, I finally found that if we arrange the stimuli in the following order, pressure-marks, X-rays, light-shock, and lamp-light, an impression of any one of them can be reversed by subsequent exposure to any other following it in the list, but under no circumstances by any one preceding it. For example, pressure-marks can be reversed by any of the other three stimuli, while X-ray images are only reversed by light-shock and lamp-light.

Experiments with Becquerel rays have given rather uncertain results. Pressure-marks can be reversed by them, and they in turn can be reversed by lamp-light, but these were the only two cases in which reversals were obtained, which makes it difficult to fit the rays into the series and still have the rule hold.

These experiments show that the effects of the different kinds of stimuli on the sensitive film are quite different. * J. U. Bose, Proc. Roy. Soc. June 19, 1902. 
Much more experimental work will have to be done before any definite notion can be obtained as to the nature of the changes produced by the action of radiation of any sort, and it is hoped that the experiments described in this paper may prove suggestive to others. Doubtless an exhaustive study of the action of various chemical agents on the plate between the two exposures would throw much light on the cause of the reversals.

If I interpret the strain-theory correctly, the application to these phenomena would be to assume that the light-shock produces a negative strain, while lamp-light produces a positive strain, either of which yields an image on development. The reversal in cases where the lamp-light follows the impression of the light-shock could be explained by assuming that the negative strain has to be undone before the positive strain can begin, consequently these parts of the plate lag behind the parts which have not received the light-shock. We should then have to assume that the positive strain once started can be continued by a stimulus which, acting first, would bave produced a negative strain, in order to account for the fact that exposures to lamp-light are not reversed by light-shocks. Moreover, it is difficu t to explain on the straintheory that two different stimuli acting in succession may produce only the same effect as one of them acting alone. It appears to me that the strain-theory would lead us to suppose that the negative strain produced by the first stimulus might be exactly neutralized by a stimulus which produces a positive strain, the plate returning to its original condition, i. e. not darkening on development. This is never the case.

In cases where reversed lines appear in the spectrum, which are suspected of being pho ographic in origin, $i$. e., not true absorption-lines, the following precautions should be taken. Repeat the exposure a number of times, using successively smaller diaphragms before the prism. If the reversal is ordinary solarization, due to over-exposure, it should disappear when the intensity of the light is sufficiently reduced. It seems to me that in such cases the reversed line should be bordered by bright edges, which does not seem to be the case in the photographs publisbed by Professor Trowbridge. If the Clayden effect is suspected, the source of light should be examined with a revolving mirror, to determine whether a dual illumination is present. The speed of the mirror should not be too great, otherwise the phosphorescence, if it exists, may be spread out to such an extent that no trace of it appears. This may account for the failure to obrain evidence of a dual illumination in the case of heavy discharges in quartz tubes. 
Phil. Mag. Ser. 6, Vol. 6, Pl. XXVI.

Fig. 1.

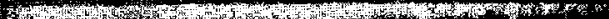

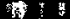

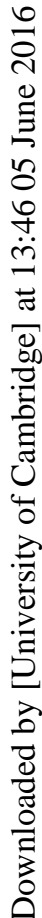
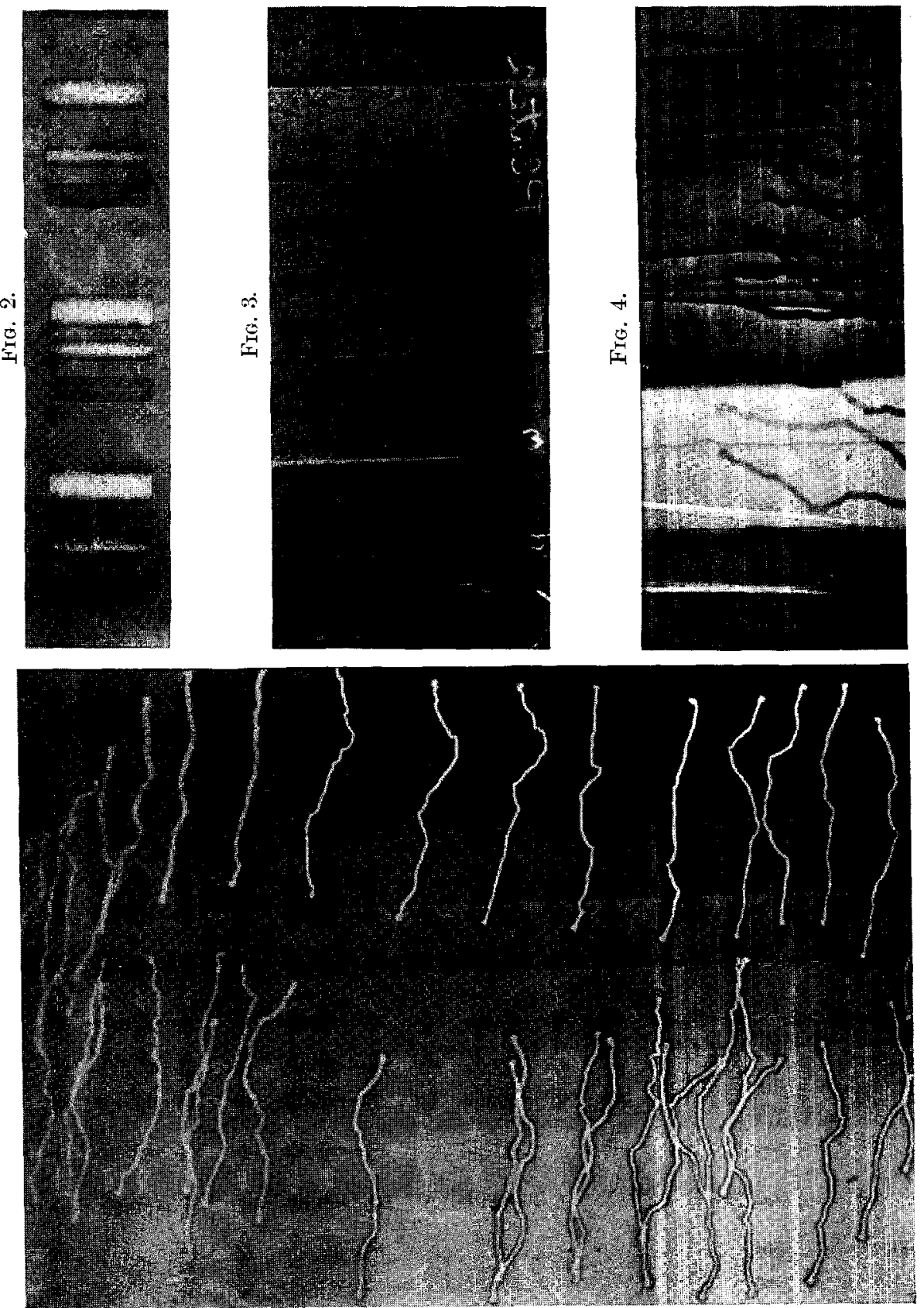

FIG. 5. 